\title{
Research and Improvement of LPDC Decoding Algorithm
}

\author{
Tingting Chen ${ }^{\mathrm{a}}$, Riuan $\mathrm{Liu}^{\mathrm{b}}$ and Lirong Diao ${ }^{\mathrm{c}}$ \\ School of electronic and communication engineering, Tianjin Normal University, Tianjin300387, \\ China \\ a13002219230@163.com, bruianliu@sina.com,cdiaolirong0716@126.com
}

Keywords: LDPC, BP algorithm, LLR BP algorithm, Tanner graph.

\begin{abstract}
Low Density Parity Check(LDPC) code itself has a good performance and the application of LDPC in the communication field is extensive. LDPC codes have the characteristics of low bit error rate, easy adjustment, low decoding complexity and excellent decoding performance. LDPC is considered to be the "best performance" in the field of coding and decoding ${ }^{[1]}$. In this paper, we mainly introduce the theory of LDPC codes. And the use of the method of the LDPC code to do a further explanation. Several simple decoding schemes of LDPC are studied. Finally, using the method of function approximation to replace the confidence function to improve the BP LLR algorithm, and simulation experiments are carried out.
\end{abstract}

\section{Introduction}

There are some differences between LDPC coding decoding and other block codes. LDPC is generally carried out by iterative decoding scheme. LDPC coding decoding make the full use of all information bits within the code word to improve the correlation between decoding accuracy, which based on consideration of the channel characteristics.

During message iterative decoding, hard decision decoding refers to the transmission of the message is a bit value, such as Bit Flipping algorithm(BF), Weighted Bit Flipping algorithm(WBF) and so on; Soft decision decoding refers to the transmission of the message that associated with posterior probability, such as Belief Propagation, BP-Based, Min-sum algorithm and so on ${ }^{[2]}$.

\section{Research on Improved Algorithm of Log Domain BP}

\subsection{Log Domain BP Decoding Algorithm.}

Probability domain BP algorithm needs to calculate a lot of parameters, the complexity of the calculation is relatively high. Log domain BP decoding algorithm is proposed for the transfer of information between nodes using the likelihood ratio, so that the original complex calculation into a simple addition, the complexity of the natural computation is reduced[3]. This use of the likelihood ratio algorithm called the log domain likelihood ratio BP algorithm, referred to as the LLR BP algorithm.

\subsection{Algorithm improvement method.}

Theoretically the number of iterations closer to the maximum value, lower error rate, but actually, a lot of time iterations did not reach maximum values when the bit error rate (BER) has stabilized, simply increasing the number of iterations and can not effectively reduce BER[4]. Usually the end of the iteration loop is determined by the test factor. In order to reduce the complexity of the algorithm, the test factor is selected as the maximum value of bit error bit number. Therefore, if the larger values of factor analysis is selected, then the number of iterations is less, it will lead to worse decoding performance; similarly, if you choose to test factor value is smaller, the number of iterations, the more, you'll get the better decoding performance. In the process of iteration, the computation complexity of the belief propagation function $\tanh (\mathrm{x} / 2)$ is relatively high, so it can reduce the computational complexity to a large extent by finding a function that can be approximated by a certain function.

If the belief propagation function is replaced by the approximation function, the simple method is to use the approximation of the rational function. Using a rational function to approximate the belief 
propagation function. Approximation of functions of order will affect approximation effect, higher order functions, approach to belief propagation function better, computation is more complex; whereas function order number is low, approach to belief propagation function effect is worse. The calculation is more simple. Therefore, the three order Taylor expansion $f 3(x)$ to approximate the function, you can get a good approximation effect, the computational complexity will not increase a lot. In addition, using $\mathrm{g}(\mathrm{x})=\tanh (\mathrm{x} / 2)-\mathrm{f} 3(\mathrm{x})$. To correct $\mathrm{f} 3(\mathrm{x})$, so that you can not significantly increase the computational complexity of the case approximation. Among them,

$$
\begin{aligned}
& \tanh (x / 2)=\frac{\exp (x / 2)-\exp (-x / 2)}{\exp (x / 2)+\exp (-x / 2)}=\frac{\exp (x)-1}{\exp (x)+1} \\
& f(x)=f_{3}(x)=1-\frac{12}{12+6 x+3 x^{2}+x^{3}}=1-\frac{12}{12+x(6+x(3+x))}
\end{aligned}
$$

Fig.1 is a modified function.

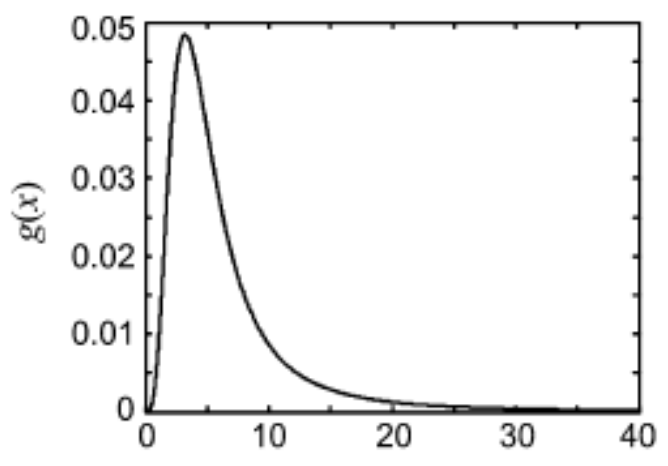

Fig. $1 \mathrm{~g}(\mathrm{x})$ function

\subsection{Performance simulation of improved algorithm.}

The performance of the BP algorithm and the improved algorithm are compared in the simulation. Hypothesis The number of bits than the original code word that is greater than 1 . The maximum number of iterations is set to 5 , that is, the maximum number of five iterations. Setting code length bit is $n=512$ bit, code rate is $R=1 / 2$, iteration number is 30 times. Height is $r=0.051$. Assuming that the channel is AWGN, using BPSK modulation mode. Performance simulation of the log domain BP algorithm has the test factor and no test factor in the performance Fig. 2 is performance simulation. Table 1 shows the number of iterations of the two algorithms in the corresponding Fig. 2.

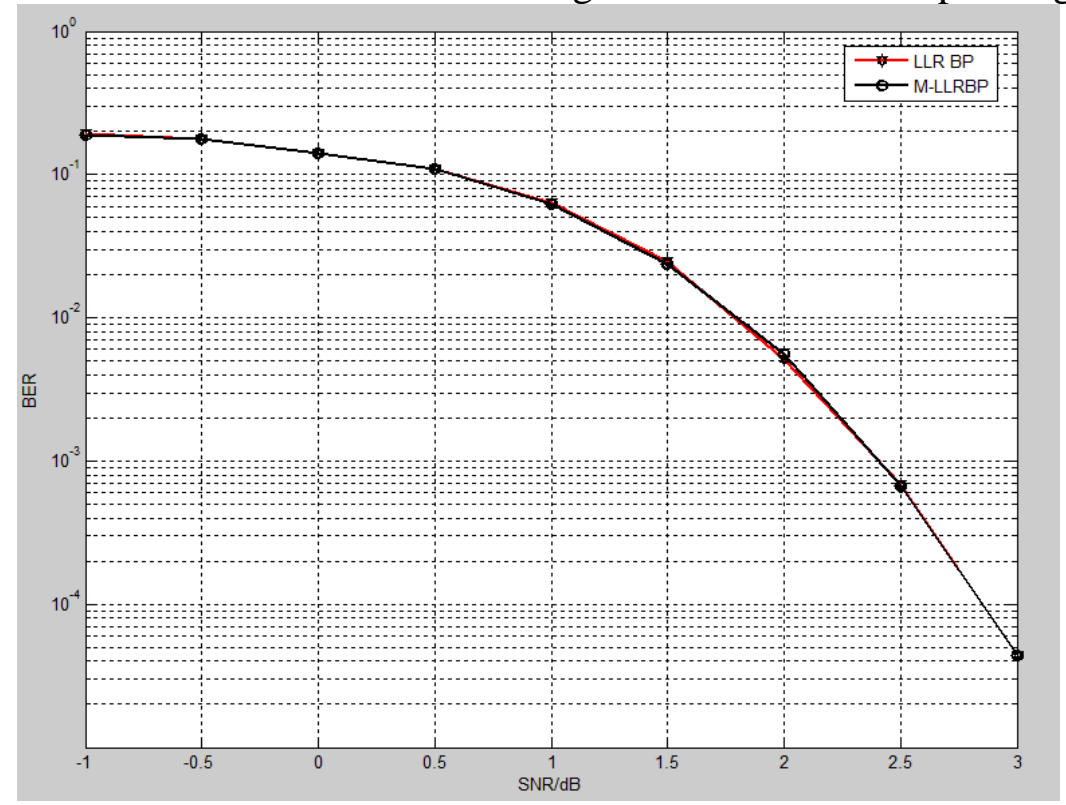

Fig. 2 Effect of test factor on performance of algorithm

In Fig. 2 LLR BP is the log domain BP algorithm, M-LLR BP represents the log domain BP algorithm with the test factor. It can be seen that the two curves are almost identical, and it is also clear that adding the test factor will not affect the decoding performance of the algorithm. 
Table 1 Comparison of iterative times of decoding algorithm

\begin{tabular}{ccc}
\hline SNR/dB & LLRBP & M-LLRBP \\
\hline-1.0 & 30.00 & 8.26 \\
-0.5 & 29.99 & 9.37 \\
0 & 29.99 & 11.72 \\
0.5 & 29.32 & 15.41 \\
1 & 25.67 & 20.04 \\
1.5 & 23.38 & 22.02 \\
2 & 23.22 & 22.84 \\
2.5 & 23.13. & 23.11 \\
\hline
\end{tabular}

Table 1 can be clearly seen in the same conditions of the signal to noise ratio, adding the test factor of the algorithm is generally smaller than the number of iterations of the algorithm does not add the iteration factor. When $\mathrm{SNR}<1.5 \mathrm{~dB}$, the number of iteration is reduced and the number of iterations of the algorithm is relatively large; when $\mathrm{SNR}>1.5 \mathrm{~dB}$, The algorithm of adding the test factor is almost identical with the BP algorithm in the log domain on the number of iterations. This is because the high SNR does not need to judge whether the test factor to achieve stability on the output of the correct decoding results. It can be concluded that the performance of the algorithm is almost identical with the classical algorithm, but the low SNR to noise ratio can reduce the number of iterations and improve the performance of the decoding. But there is no advantage in the high SNR.

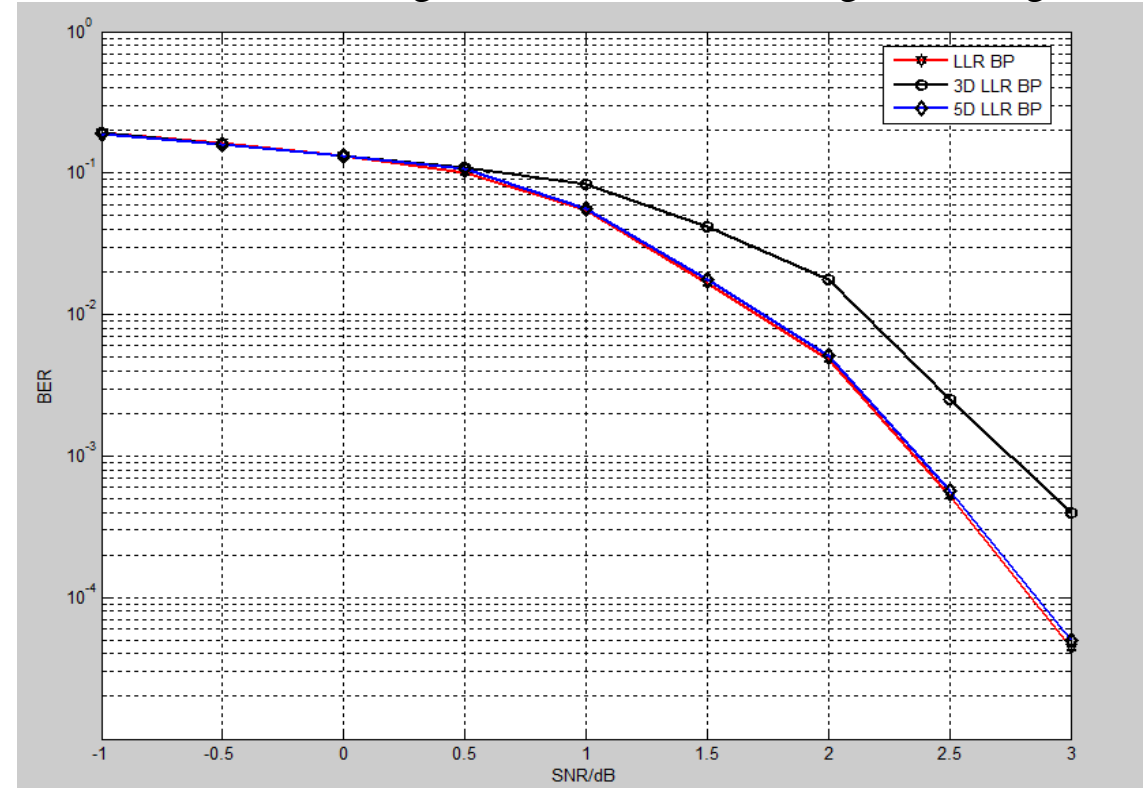

Fig. 3 Performance simulation of approximation functions with different orders

Fig. 3 shows that the performance of the five order Taylor expansion is almost the same as that of the original log domain BP algorithm by using the method of function approximation. And the performance of the three order function approach is slightly worse than the other two algorithms. But the five order expansion of the approximation function increases the computational complexity to some extent.

After the approximation function is modified, the performance of the three order expansion and other algorithms are compared. 


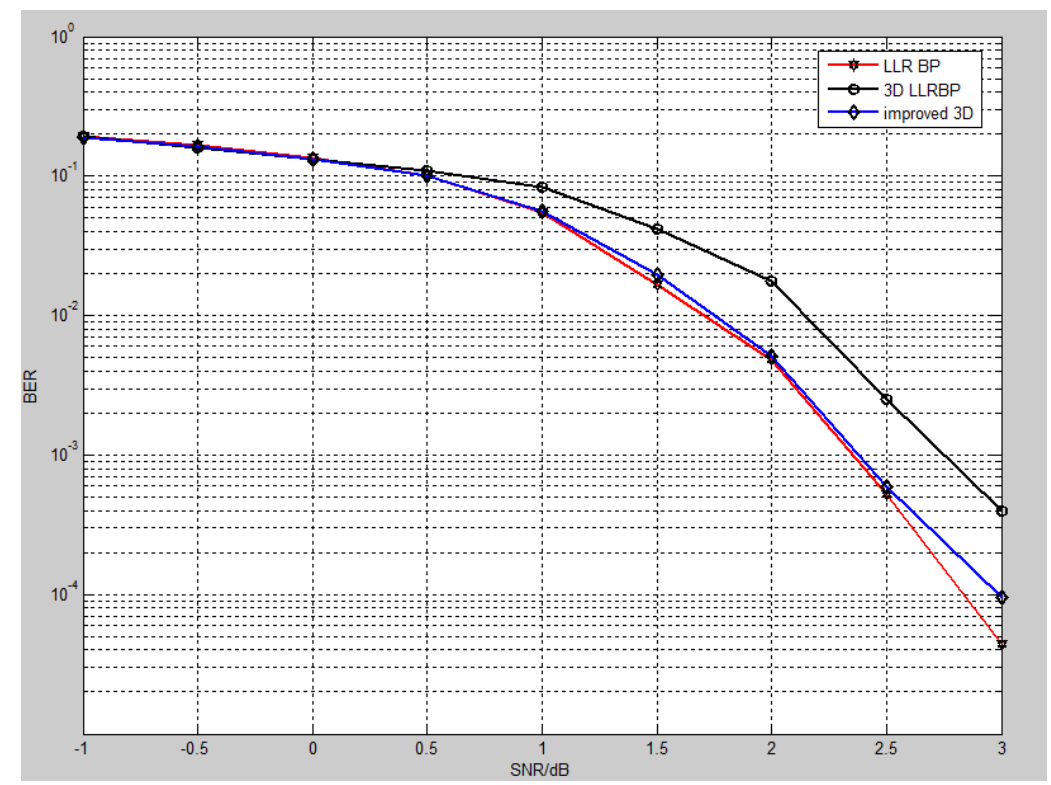

Fig. 4 Performance comparison of confidence function for 3 order and 3 order improvement

Fig. 4 can be seen that the use of the function of the three order approximation function is modified, the modified three - order approximation function performance is significantly better than the three order approximation. The performance of the modified algorithm is very close to the performance of the log domain BP algorithm.

Correction of the third order approximation function of the algorithm improved, in performance and classical LLR BP algorithm are basically the same, slightly lower calculation complexity, the number of iterations significantly reduced, so the algorithm without increasing the complexity of the algorithm, reducing the decoding complexity, and reduce the decoding time.

\section{Summary}

In this paper, the basic theory of LDPC code is studied from the definition of LDPC and the Tanner diagram. In addition, the BP decoding, log domain BP algorithm and other algorithms are deeply studied, and the performance of these algorithms is simulated and analyzed.

According to the theory of LLR BP decoding algorithm optimization, through the method of function approximation improved the log domain BP algorithm, the confidence function is carried out on the three order Taylor expansion and correction, and then the performance simulation and analysis of this algorithm. The performance of the proposed algorithm is similar to that of the classical log domain BP algorithm, but the number of iterations is obviously reduced under the condition of low noise. That is to say, the complexity of the algorithm is reduced.

\section{Acknowledgments}

This research was supported by the Tianjin natural science fund (13JCYBJC15800).

\section{References}

[1] J. Hou. P. H. Siegel and L. B. Milstein. Performance Analysis and Code Optimization of Low Density Parity Check Codes on Rayleigh Fading Channels.IEEE Journal on Selected Areas in communications , Vol 19, May 2001.

[2] D.J. MacKay and R.M. Neal. Near Shannon limit performance of low density parity check codes. Electronics Letters, Vol. 32,no. 18,pp. 1645,1996.

[3] T. Richardson and R. Urbanke. The capacity of low-density parity check codes under message-passing decoding[J].IEEE Trans. Information Theory, 2001,Vol. 47,no. 2, pp. 599-618.

[4] Y. Nana,E. Sharon, and S. Litsyn. Improved decoding of LDPC coded modulations [J]. IEEE Communications Letters,2006, Vol,10,no. 5,375-377. 